\title{
Zehn Jahre Hoher Vertreter - Lehren für die EU-Außen- und Sicherheitspolitik nach Lissabon
}

\author{
Gisela Müller-Brandeck-Bocquet und Carolin Rüger**
}

Gut ein Jahr nach dem Abtreten des ersten Hohen Vertreters der Europäischen Union für die Gemeinsame Außen- und Sicherheitspolitik (HV), Javier Solana, und der Wahl Catherine Ashtons zu seiner Nachfolgerin in einem durch den Vertrag von Lissabon substanziell veränderten und aufgewerteten Amt bietet es sich an, einen bilanzierenden Blick auf die Dekade Solana von 1999 bis 2009 zu werfen. Eine solche Bilanz ist vor allem dann für die Integrationsforschung von Interesse, wenn sie auch aufzuzeigen vermag, inwiefern der erste HV die EU-Außen- und Sicherheitspolitik geprägt und vorangebracht hat und inwiefern die Neuerungen des Primärrechts, insbesondere der doppelhütige „HV 2.0“1 sowie der Europäische Auswärtige Dienst (EAD) ,lessons learned“ aus den Erfahrungen und Erfolgen, aber auch aus den Defiziten der Ära Solana darstellen.

\section{Amt, Rollenverständnis und Persönlichkeit des ersten HV}

Ein Rückblick auf die Dekade Solana steht zwangsläufig unter der übergeordneten Fragestellung, inwieweit der erste HV das außen- und sicherheitspolitische Profil der Europäischen Union stärken konnte. Welche Impulse vermochte er der Gemeinsamen Außen- und Sicherheitspolitik (GASP) und der Europäischen Sicherheits- und Verteidigungspolitik (ESVP) zu verleihen, welche Prioritäten setzte er und wie konnte er beide Politikfelder zusammen mit den Mitgliedstaaten und anderen wichtigen Akteuren gestalten? Welches Vermächtnis hinterlässt Solana, der auf der internationalen Bühne als Gesicht und Stimme Europas galt, seiner Nachfolgerin?

\section{Ein Amt ist, was man daraus macht ${ }^{2}$}

Alle Autoren des Tagungsbandes ${ }^{3}$ stimmen darin überein, dass der erste HV aus seinem Amt bei Weitem mehr gemacht hat als der Vertrag von Amsterdam vorgesehen hatte. In der Tat bestimmte Art. 26 EUV-Amsterdam ${ }^{4}$ den Generalsekretär des Rates zum HV, wies ihm jedoch nur unterstützende Aufgaben bei der „Formulierung, Vorbereitung und Durchfüh-

* Prof. Dr. Gisela Müller-Brandeck-Bocquet, Professur für Europaforschung und Internationale Beziehungen, Julius-Maximilians-Universität Würzburg.

Carolin Rüger, Wissenschaftliche Mitarbeiterin an der Professur für Europaforschung und Internationale Beziehungen, Julius-Maximilians-Universität Würzburg.

Der Beitrag stützt sich auf den Sammelband, der im Rahmen eines von der Europäischen Kommission geförderten Forschungsprojekts entstand. Vgl. den in Kürze erscheinenden Tagungsband: Gisela Müller-BrandeckBocquet/Carolin Rüger (Hrsg.): The High Representative for the EU Foreign and Security Policy - Review and Prospects, Baden-Baden 2011, im Erscheinen.

1 Carolin Rüger: A position under construction: Future prospects of the High Representative after the Treaty of Lisbon, in: Müller-Brandeck-Bocquet/Rüger: The High Representative for the EU Foreign and Security Policy, 2011.

2 Claudia Major: Pulling the strings behind the scenes - but never against the member states: Solana's role in the launch of EU military operations, in: Müller-Brandeck-Bocquet/Rüger: The High Representative for the EU Foreign and Security Policy, 2011.

3 Müller-Brandeck-Bocquet/Rüger: The High Representative for the EU Foreign and Security Policy, 2011.

4 Vertrag über die Europäische Union in der Fassung des Vertrags von Amsterdam, in: Amtsblatt der EU, Nr. C 340 vom 10. November 1997. 
rung" der politischen Entscheidungen im Bereich der GASP zu. Solana aber gelang es sehr bald, die engen Fesseln dieser dienenden Funktion zu sprengen. Dies lag zum einen an seiner Persönlichkeit und seinen ,impressive leadership credentials" ${ }^{\text {" }}$. Es ist zu betonen, dass mit der Wahl Solanas, des spanischen Sozialisten, der von 1992 bis 1995 Außenminister seines Landes und von 1995 bis 1999 NATO-Generalsekretär gewesen war, die EU-Staats- und Regierungschefs sich 1999 bewusst für einen vergleichsweise starken HV entschieden. ${ }^{6}$ Dabei lieferten die internationale Lage und die neuen sicherheitspolitischen Herausforderungen die Hintergrundmatrix, die es dem ersten HV erlaubten, sein Amt weit über die Bestimmungen des Vertrags von Amsterdam hinaus aufzuwerten und auszubauen; denn angesichts des zeitgleich ablaufenden Kosovo-Konflikts waren die EU-Mitgliedstaaten Ende der 1990er Jahre endlich dazu bereit, ihre sicherheitspolitische Abstinenz aufzugeben und den Einstieg der Europäischen Union in den Aufbau operativer militärischer und ziviler Kriseninterventionskräfte zu wagen. Damit bot insbesondere die ESVP dem HV die Chance, die Europäische Union weltweit als nützlichen Akteur ins Spiel zu bringen und sich selbst als Europas Chefdiplomat und Krisenmanager zu profilieren.

Solana beanspruchte somit eine wichtige und herausgehobene Rolle für sein Amt. Einerseits wusste der international erfahrene Politiker zwar, dass er die in GASP und ESVP weitestgehend allein entscheidungsbefugten Mitgliedstaaten nicht verprellen durfte und ihre Souveränität zu respektieren hatte. Andererseits jedoch wollte der selbstbewusste HV mit den ganz Großen der internationalen Szene an einem Tische sitzen und Weltpolitik gestalten. Zu solch einem Rollen- und Selbstverständnis befähigten ihn sein außenpolitisches Renommee sowie seine außerordentlich gute Vernetzung mit dem , who is who" der internationalen Politik, mit welchem er persönlich freundschaftliche Beziehungen pflegte. Dabei taten sein Politikstil sowie seine charmante, warmherzige und spontane Art ein Übriges. Dass ihm all dies eine weit über die Grenzen seines Amtes hinausreichende politische Bedeutung verschaffte, betonen nahezu alle Autoren des Sammelbandes.

\section{Dienste und Strukturen}

Bei der Frage „Was bleibt von der Dekade Solana?“ geraten vorrangig die Dienste und Strukturen in den Blick, die der erste HV schuf und mit deren Hilfe er seine Rolle in und seinen Einfluss auf GASP und ESVP geltend machen und implementieren konnte. Hier handelt es sich ohne Zweifel um ein zentrales und bleibendes Vermächtnis Solanas, denn diese Dienste und Strukturen sind inzwischen allesamt in den neu aufgebauten EAD überführt worden (siehe unten).

Ohne seine Dienste hätte Solana seine Rolle nicht in dem eben beschriebenen expansiven Sinne ausüben können. Deren rascher Aufwuchs zeigt sich bereits rein numerisch: Während Solana bei Amtsantritt über rund 70 Mitarbeiter verfügte, arbeiteten ihm zehn Jahre später zwischen 600 und 700 Mitarbeiter in Brüssel direkt zu. ${ }^{7}$ Trotz aller Vorbehalte, die die Mitgliedstaaten und insbesondere die Kommission dem Amt des HV anfangs entgegenbrachten, trugen beide den Auf- und Ausbau seiner Dienste mit. Denn sie hatten rasch erkannt, dass sie angesichts des internationalen Kontextes eine Figur wie den HV dringend brauchten. ${ }^{8}$

5 Major: Pulling the strings behind the scenes, 2011.

6 Elfriede Regelsberger: The High Representative for the Common Foreign and Security Policy - Treaty provisions in theory and in practice 1999-2009, in: Müller-Brandeck-Bocquet/Rüger: The High Representative for the EU Foreign and Security Policy, 2011.

7 Hylke Dijkstra: Solana and his Civil Servants: An Overview of Political-Administrative Relations, in: MüllerBrandeck-Bocquet/Rüger: The High Representative for the EU Foreign and Security Policy, 2011.

8 Fraser Cameron: The EU's External Action Service - Golden or Missed Opportunity?, in: Müller-BrandeckBocquet/Rüger: The High Representative for the EU Foreign and Security Policy, 2011. 
Darum konnte Solana seine Mitarbeiterstäbe rasch aufbauen. Konkret angesprochen sind hier die fünf Dienste, die direkt unter der Autorität des HV standen und die fortlaufend dem Gang von GASP und ESVP angepasst wurden: die 1999 geschaffene Analyse- und Frühwarneinheit (Policy Unit, PU); das Joint Situation Centre of the European Union (SitCen), das 2003 zur Informationsgewinnung vor ESVP-Einsätzen eingerichtet wurde; der 2001 geschaffene Europäische Militärstab (EU Military Staff, EUMS); die 2007 beschlossene und als ziviles Gegengewicht zum EUMS geschaffene Civilian Planning and Conduct Capability (CPCC); sowie das im Aufbau befindliche Crisis Management and Planning Directorate (CMPD), das in Zukunft zum zivil-militärischen Hauptquartier der Europäischen Union ausgebaut werden könnte. Diese Dienste erarbeiteten zum einen Informationen und Lageberichte, die in den außen- und sicherheitspolitischen Entscheidungsprozess eingingen, zum anderen waren sie in den Implementierungsprozess der zivilen und militärischen ESVP-Missionen eingebunden und hier von besonderer Nützlichkeit. ${ }^{9}$ Zum Apparat des HV müssen auch die Sonderbeauftragten der Europäischen Union (EU Special Representatives, EUSRs) gezählt werden, die der erste HV mit großem diplomatischen Geschick sukzessive in sein Umfeld zu integrieren verstand. ${ }^{10}$

Obgleich der Aufbau dieser Dienste ohne jeden Zweifel eines der herausragenden Verdienste Solanas darstellt, wird bisweilen der organisatorische Wildwuchs kritisiert, in welchem sie entstanden. Solana wird vorgeworfen, unsystematisch und ohne strategische Blaupause vorgegangen zu sein. ${ }^{11}$ Dem lässt sich entgegenhalten, dass die ESVP-Strukturen jeweils in Reaktion auf konkrete Ereignisse und neue Anforderungen an die Europäische Union entstanden, was strategisch weitblickende Planung erschwert. Als sicher kann jedoch angenommen werden, dass administrative Sorgfalt nicht die Sache des als chaotisch geltenden Solana war.

\section{Der Hohe Vertreter und weitere Schlüsselakteure in Europas Außen- und Sicher- heitspolitik}

Trotz der Aufwertung, die Solana seinem Amt verschaffen konnte, bleibt zutreffend, dass der HV mitnichten der einzige und schon gar nicht der wichtigste Akteur in der Gestaltung und Ausführung der EU-Außen- und Sicherheitspolitik war. So gehört zu den Gemeinplätzen der EU-Außenpolitikforschung, dass das Zusammenspiel zwischen Solana und den anderen GASP- und ESVP-Akteuren oft kompetitiver, ja konfliktiver Natur war.

\section{Die Beziehungen des $H V$ zu den Mitgliedstaaten}

Zweifelsohne stellten die Mitgliedstaaten als die nach wie vor wichtigsten Akteure in der intergouvernemental geprägten GASP und ESVP die zentrale Begrenzung der Gestaltungsmacht und des Einflusses des HV dar. Denn sie entschieden letztlich, wie weit Solanas Rolle reichen konnte. So tendieren in GASP und ESVP alle Mitgliedstaaten dazu, einen zwar von ihnen mandatierten, aber „brüsselisierten“ Akteur an die kurze Leine zu nehmen und unter Kontrolle zu halten. ${ }^{12}$

9 Simon Duke: Under the Authority of the High Representative, in: Müller-Brandeck-Bocquet/Rüger: The High Representative for the EU Foreign and Security Policy, 2011.

10 Cornelius Adebahr: From Competitors to Deputies: How the EU Special Representatives Integrated into the Solana System, in: Müller-Brandeck-Bocquet/Rüger: The High Representative for the EU Foreign and Security Policy, 2011.

11 Dijkstra: Solana and his Civil Servants, 2011; Duke: Under the Authority of the High Representative, 2011.

12 Rikard Bengtsson/David Allen: Exploring a triangular drama: The High Representative, the Council Presidency and the Commission, in: Müller-Brandeck-Bocquet/Rüger: The High Representative for the EU Foreign and Security Policy, 2011. 
Solana war sich der Grenzen, die ihm in Gestalt der Mitgliedstaaten gesetzt waren, vollkommen bewusst. Schnell hatte er erkannt, dass er stark nur mit den Mitgliedstaaten sein konnte, niemals gegen sie. ${ }^{13}$ In Erkenntnis, dass letztlich die Mitgliedstaaten die GASP- und ESVP-Entscheider sind, begegnete er ihnen stets mit Respekt und interpretierte seine Rolle als die eines ,facilitator', der die Entschluss- und Handlungsbereitschaft der Mitgliedstaaten befördern musste. Besonders dann, wenn sich unter ihnen noch keine klare, einheitliche Positionierung abzeichnete, konnte ,facilitator' Solana durch seinen vorsichtigen, überzeugungsorientierten Arbeitsstil erfolgreich Entscheidungen vorbereiten und ermöglichen. Doch wenn sich keinerlei gemeinsame Interessensschnittmengen finden ließen, tendierten Solanas Handlungsmöglichkeiten gegen Null. Hierfür stehen exemplarisch die krassen Meinungsdivergenzen der Mitgliedstaaten im Falle des Irak, die den Tiefpunkt der Beziehungen zwischen ihnen und dem HV markierten.

Solanas Aussichten, die Europäische Union zu einem geschlossenen und entschiedenen Handeln in internationalen Krisen und Konflikten zu bewegen, waren immer dann besonders groß, wenn einer oder mehrere große Mitgliedstaaten zum Handeln bereit waren. Dabei kam es durchaus auch vor, dass Mitgliedstaaten sich des HV bedienten, um ihren eigenen außenpolitischen Anliegen durch diese Europäisierung eine höhere Legitimation zu verschaffen und europäisches ,burden-sharing ' zu erreichen, wie beispielsweise 2003 geschehen, als Frankreich sich für eine militärische EU-Mission im Kongo einsetzte. Dies gab Solana dann die Möglichkeit, aktiv auch andere Mitgliedstaaten für gemeinsames Handeln zu gewinnen.

Wenn die Mitgliedstaaten sich manchmal also Solanas bedienten, um eigene Interessen und Prioritäten durchzusetzen, so griff auch der HV bisweilen auf diese Taktik zurück. In Kenntnis des Reflexes der Mitgliedstaaten, ihre außen- und sicherheitspolitische Souveränität eifersüchtig zu wahren, verzichtete Solana meist darauf, eigene Ideen direkt vorzubringen. Vielmehr versuchte er, sie den Mitgliedstaaten - insbesondere der amtierenden Ratspräsidentschaft - zu , verkaufen'. Derart erarbeitete er sich quasi ein außen- und sicherheitspolitisches Initiativrecht, was mitnichten im Vertrag von Amsterdam angelegt war. ${ }^{14}$ Kurz: Solana verstand es, mit den Mitgliedstaaten umzugehen, vor allem mit den Großen. Doch während manche ihm mangelnde Entscheidungskraft, gar Schwäche im Angesicht der Macht der Mitgliedstaaten vorwerfen, interpretieren andere dies als kluge Zurückhaltung und strategische Finesse: Weil Solana um die Grenzen seines Amtes und seiner Rolle wusste, vermied er offene Konfrontationen mit den Mitgliedstaaten, die ja letztlich die Entscheider waren und verlegte sich notfalls auf das Agieren hinter den Kulissen - ein Metier, das er perfekt beherrschte.

\section{Der HV und die rotierenden Ratspräsidenten}

Das Verhältnis zwischen dem HV und den jeweiligen Ratspräsidentschaften (immerhin 21 an der Zahl während Solanas Amtszeit) kann als Sonderfall der Beziehungen des HV zu den Mitgliedstaaten verstanden werden. Dabei lassen sich interessante Beziehungsmuster erkennen. ${ }^{15}$ Auf der einen Seite ist das Verhältnis zwischen HV und Präsidentschaft von gleichbleibenden Parametern bestimmt - unabhängig vom jeweiligen Ratsvorsitz. So neigen alle Präsidentschaften dazu, den HV unter Kontrolle halten und damit die mitgliedstaatlichen Befugnisse wahren zu wollen. Auf der anderen Seite lassen sich durchaus Unterschiede

13 Major: Pulling the strings behind the scenes, 2011.

14 Regelsberger: Treaty provisions in theory and in practice 1999-2009, 2011.

15 Bengtsson/Allen: Exploring a triangular drama, 2011. 
in den Beziehungen und der Zusammenarbeit zwischen dem HV und den verschiedenen Vorsitzen beobachten. In dieser Varianz spielen Größe, politisches Gewicht, außen- und sicherheitspolitisches Selbstverständnis und Gestaltungswille der jeweiligen Präsidentschaft die entscheidende Rolle. Wenn große Mitgliedstaaten den Ratsvorsitz stellen, wirken sich deren finanzielle und personelle Ressourcen zumeist restriktiv auf den Handlungsspielraum des HV aus. Ausnahmen bestätigen aber auch hier die Regel. So wird der deutschen Ratspräsidentschaft 2007 bescheinigt, sehr gut mit Solana zusammengearbeitet zu haben. Schlechter ausgestattete, das heißt meist kleine Mitgliedstaaten neigen in der Regel zu enger Kooperation mit dem HV. Gleiches gilt für neue Mitgliedstaaten.

Einen großen Einfluss auf das Verhältnis zwischen HV und Ratspräsident hatte - angesichts des Politikstils Solanas wenig überraschend - auch die persönliche Beziehung zwischen ihm und dem Führungspersonal des betreffenden Staates. Solana richtete seinen Fokus dabei möglicherweise zu sehr auf die Ebene der Staats- und Regierungschefs und vernachlässigte tendenziell die Außenminister. Außerdem hing die Frage nach Solanas Einfluss nicht zuletzt davon ab, welche Thematik zu behandeln war und welche Region Priorität genoss. Da Solanas Fokus vor allem auf dem westlichen Balkan und dem Nahen Osten lag, konnten Vorsitze, die Initiativen beispielsweise in Afrika oder Lateinamerika vorantreiben wollten, dies relativ ,ungestört“ vom HV tun. ${ }^{16}$

Insgesamt konnte der Vollblutdiplomat Solana auch seine Arbeitsbeziehungen zu den verschiedenen Ratsvorsitzen positiv gestalten. Dennoch bewertete er die rotierende Präsidentschaft als ,,a thing of yesterday and not of tomorrow"17 - und teilte damit die Ansicht vieler Beobachter, dass sich die Rotation sowohl auf die interne Dynamik des EU-Systems als auch auf das außenpolitische Profil der Union negativ auswirkt. Es dürfte den ersten HV befriedigen, dass mit dem Vertrag von Lissabon die Bedeutung der rotierenden Präsidentschaft für Europas Außen- und Sicherheitspolitik substanziell reduziert wurde - die im Vertrag über eine Verfassung für Europa (Verfassungsvertrag) ${ }^{18}$ und im neuen Vertrag gewählte Lösung des Doppelhuts lehnte Solana allerdings eindeutig ab. Die Konstruktion und Aufgabenfülle des neuen Amtes bezeichnete er als „mission impossible“. 19

\section{HV und Kommissar für Außenbeziehungen}

Angesichts der Mehrdimensionalität des EU-Außenhandelns, das zwischen der Sphäre der Mitgliedstaaten und des Rates einerseits sowie der Sphäre der Kommission andererseits aufgeteilt ist, können Stellung und Einfluss des HV nur sinnvoll analysiert werden, wenn auch seine Arbeitsteilung mit und Zuständigkeitsabgrenzung zur Kommission berücksichtigt werden. Diese Mehrdimensionalität steht im Zentrum der horizontalen Kohärenzprobleme der Europäischen Union ${ }^{20}$ und ist Folge der Entscheidung des Vertrags von Maastricht, die EU-Außenpolitik auf die verschiedenen Säulen aufzuteilen.

Folglich war die Kommission beziehungsweise der Kommissar für Außenbeziehungen der strukturell bedingte Gegenspieler des HV; zumal im Bereich des zivilen Krisenmanagements, für welches mit der Schaffung der Europäischen Sicherheits- und Verteidigungspolitik (ESVP) nun auch die Ratsseite und damit der HV an herausgehobener Stelle zuständig waren. So ist nicht verwunderlich, dass die Beziehung zwischen HV und Außenkommissar

16 Ebenda.

17 Solana zitiert nach ebenda.

18 Vertrag über eine Verfassung für Europa, in: Amtsblatt der EU, Nr. C 310 vom 16. Dezember 2004, S. 1-474.

19 Rüger: A position under construction, 2011.

20 Ebenda. 
prinzipiell als besonders heikel gilt. ${ }^{21}$ Gleichzeitig aber erforderte die mehrdimensionale Struktur des EU-Außenhandelns enge Kooperation zwischen der (ehemals) zweiten Säule und dem Gemeinschaftsbereich; zum einen um Kohärenz herzustellen, zum anderen weil es angesichts des sehr knappen GASP-Budgets schlichtweg notwendig war, auf die reicher gefüllten Gemeinschaftskassen zurückzugreifen, verfügte der Außenkommissar doch über so „useful things like money“22.

HV Solana hatte es in seiner doppelten Amtszeit mit zwei Kommissaren für Außenbeziehungen zu tun, mit dem international renommierten und sich überparteilich gebenden Chris Patten aus Großbritannien (1999-2004) und mit Benita Ferrero-Waldner, die direkt aus dem politischen Tagesgeschäft als Österreichs Außenministerin (Österreichische Volkspartei) 2004 nach Brüssel wechselte. Obwohl Solana und Patten bisweilen ein allzu harmonisches Bild ihrer Zusammenarbeit zeichneten und Spannungen sowie Rivalitäten vornehm übergingen, bleibt festzuhalten, dass ihr Verhältnis deutlich besser war als das zwischen Solana und Ferrero-Waldner. Letzteres stand von Anfang an unter keinem guten Stern, wurde Solana doch zeitgleich mit Ferrero-Waldners Amtsantritt vom Europäischen Rat zum ersten Außenminister der Union - wie vom Verfassungsvertrag vorgesehen - designiert. Damit verkörperte er von Anbeginn eine ernsthafte Bedrohung der Zuständigkeiten und Gestaltungsbefugnisse der Außenkommissarin. Entsprechend begrenzt war die Kooperation zwischen beiden. $^{23}$

Hier ist auch auf den deutlich unterschiedlichen Machtanspruch zu verweisen, den die Kommissionspräsidenten Romano Prodi und José Manuel Barroso in den EU-Außenbeziehungen erhoben. Während Prodi die Koordination der Kommissionsportfolios mit externem Bezug (vor allem Außenhandel, Entwicklungshilfe, Erweiterung) seinem Kommissar für Außenbeziehungen Patten überließ und ihn somit zum wichtigsten Ansprechpartner Solanas machte, riss Barroso, der sich zunehmend zum ,Alphatier' der auswärtigen Beziehungen der Kommissionssphäre aufschwang, ${ }^{24}$ die Zügel spürbar an sich.

\section{Der HV und das Europäische Parlament}

Obwohl das Europäische Parlament mit Sicherheit nicht zu den Hauptakteuren der EUAußen- und Sicherheitspolitik gehört, dürfen die Beziehungen zwischen ihm und dem ersten HV nicht gänzlich unerwähnt bleiben. In Art. 21 EUV-Nizza ${ }^{25}$ kommt die deutlich untergeordnete außen- und sicherheitspolitische Rolle des Europäischen Parlaments klar zum Ausdruck. Hier werden ihm lediglich Konsultations- und Informationsrechte sowie das Recht, Fragen zu stellen und Empfehlungen abzugeben, eingeräumt. Aufgrund seiner Haushaltsbefugnisse, die mit dem Vertrag von Lissabon noch deutlich ausgeweitet wurden, hat das Europäische Parlament aber gleichwohl ein gewisses Mitspracherecht, insbesondere an der Gestaltung des kommissionsseitigen Außenhandelns der Europäischen Union.

Nachdem das Europäische Parlament sich anfangs gegen die Schaffung des Amtes des HV gestellt hatte und stattdessen für den Ausbau der Zuständigkeiten des Kommissars für Außenbeziehungen eingetreten war, versuchte es mit diversen Vorstößen, sich via HV verstärkte Mitspracherechte in der GASP zu sichern - beispielsweise mit der Forderung, Solana vor seiner Ernennung einer Anhörung zu unterziehen. Weiterhin verlangte das Europäische

21 Regelsberger: Treaty provisions in theory and in practice 1999-2009, 2011.

22 Chris Patten: Not quite the diplomat. Home truths about world affairs, London 2005, S. 159.

23 Bengtsson/Allen: Exploring a triangular drama, 2011.

24 Rüger: A position under construction, 2011.

25 Vertrag über die Europäische Union in der Fassung des Vertrags von Nizza, in: Amtsblatt der EU, Nr. C 321 E vom 29. Dezember 2006. 
Parlament, dass Solana ihm regelmäßig Rede und Antwort steht. Dem kam der HV im Zeitverlauf immer bereitwilliger nach. ${ }^{26}$ Dies erklärt sich dadurch, dass die Präsidentschaften ihre aus Art. 21 EUV-Nizza entstehenden Informationspflichten gerne an das Ratssekretariat beziehungsweise den HV delegierten. Mit dem Vertrag von Lissabon ist nun der HV offizieller Hauptansprechpartner des Europäischen Parlaments (Art. 36 EUV $^{27}$ ). Für die Dekade Solana muss jedoch klar gesehen werden, dass der erste HV dem Europäischen Parlament mehr aus Höflichkeit denn aus formaler Verpflichtung entgegenkam; ${ }^{28}$ letztendlich hielt Solana nämlich das Europäische Parlament auf Distanz, sodass es keinerlei informelle Macht gegenüber dem HV gewinnen konnte. ${ }^{29}$ Vielmehr boten seine Auftritte vor den Abgeordneten dem HV eine willkommene Gelegenheit zu zusätzlicher Profilierung von Amt und Person.

\section{Solanas Verständnis von der internationalen Rolle der Europäischen Union}

Der HV Solana sah seine Aufgabe vorrangig darin, die Stimme Europas auf der internationalen Ebene vernehmbar zu machen. Auch persönlich war er von der ,,notwendigen Rolle der EU in allen wichtigen internationalen Fragen“ überzeugt, so seine langjährige Vertraute und Sprecherin Cristina Gallach. ${ }^{30}$ Damit sah er auch die Europäische Union in die Pflicht genommen, eine größere, mehr als nur ökonomisch geprägte Rolle auf der Weltbühne zu spielen. „We have no choice, we must play a role. “31 Diese Grundüberzeugung machte aus dem durchaus transatlantisch ausgerichteten Solana einen glaubhaften Verfechter eines international starken, autonom handlungsfähigen Europas.

Als Gesicht und Stimme einer Weltmacht im Werden verfocht der erste HV zudem die Auffassung, dass die Gestaltung der EU-Außenpolitik die Chance und Notwendigkeit zur Konzeption einer neuartigen Außenpolitik beinhalte: „We need to think differently about foreign policy as such. Foreign policy these days should not be just about diplomats, soldiers and development workers. [...] Modern foreign policy should be broader and involve wider sets of people. [...] If the European Union gets its act together on energy, climate change and migration, we will have created the building blocks for a foreign policy fit for the $21^{\text {st }}$ century." So zitiert Franco Algieri ${ }^{32}$ Solanas Vorstellung einer konzeptionell neuen Außenpolitik, die sich aktiv auf die Globalisierung und zunehmende weltweite Interdependenzen einstellt. Solana war angesichts des fundamentalen Umbruchs in den internationalen Beziehungen davon überzeugt, dass kein Staat - und sei er auch noch so mächtig - alleine die anstehenden Probleme würde lösen können; vielmehr sah er intensive internationale Zusammenarbeit und einen ausgeprägten Multilateralismus als zielführend an.

In seiner Analyse des Außenpolitikansatzes Solanas und dessen Vorstellung von der internationalen Rolle der Europäischen Union betont Algieri, dass beides sich in kondensierter Form in der ersten Europäischen Sicherheitsstrategie (ESS) „A secure Europe in a better world" von $2003^{33}$ wiederfindet, an deren Konzeption Solana federführend beteiligt war und

26 Regelsberger: Treaty provisions in theory and in practice 1999-2009, 2011.

27 Vertrag über die Europäische Union in der Fassung des Vertrags von Lissabon, in: Amtsblatt der EU, Nr. C 83 vom 30. März 2010, S. 13-45.

28 Ben Crum: Parliamentarization of the CFSP through informal institution-making? The fifth European Parliament and the EU High Representative, in: Journal of European Public Policy 3/2006, S. 383-401, hier S. 394.

29 Ebenda, S. 397.

30 Hintergrundgespräch mit Cristina Gallach am 18.03.2010 in Brüssel.

31 Solana zitiert nach Bengtsson/Allen: Exploring a triangular drama, 2011.

32 Franco Algieri: Understanding differences: An attempt to describe Javier Solana's view of the EU's international role, in: Müller-Brandeck-Bocquet/Rüger: The High Representative for the EU Foreign and Security Policy, 2011.

33 Ein sicheres Europa in einer besseren Welt. Europäische Sicherheitsstrategie, Brüssel 12.12.2003. 
die weitgehend seine Handschrift trägt. In der ESS verpflichtet sich die Europäische Union dazu, zur Sicherheit in allen Teilen der Welt beizutragen, vorrangig aber für Frieden und Stabilität in ihrem unmittelbaren Umfeld zu sorgen. Die Übernahme internationaler Verantwortung kann in ultima ratio auch militärische Interventionen bedeuten. Insgesamt setzt die ESS auf intensive internationale Zusammenarbeit und bekennt sich offen zu einem ausgeprägten Multilateralismus. Schließlich folgt die ESS einem weiten Sicherheitsbegriff, der neue Bedrohungen berücksichtigt, und tritt für ein breites, ganzheitliches Vorgehen ein, dessen markantester Ausdruck inzwischen der doppelte zivil-militärische Ansatz der ESVP ist, bekanntermaßen das ,Markenzeichen“ des europäischen Krisenmanagements. ${ }^{34}$ Doch trotz ihrer operativen ESVP-Kapazitäten bleibt die Europäische Union eine zuvörderst zivile und normative Macht, die vorrangig mittels Dialogen und Verhandlungen agiert. ${ }^{35}$ Dies sei der „European way“ zur internationalen Ordnung beizutragen, schreibt Gallach ${ }^{36}$ und deutet damit implizit an, dass Solana sowohl Miturheber als auch überzeugter Verfechter dieser europäischen Methode in der Praxis war.

Auch in der überarbeiteten ESS aus dem Jahr 2008 findet sich dieses sehr deutlich von Solana mitgeprägte und mitgetragene Selbstverständnis der EU-Außen- und Sicherheitspolitik wieder. Schließlich enthält das überarbeitete Dokument Forderungen nach strategischem Denken und kohärentem, entschlossenem Handeln der Europäischen Union. So schließt die ESS aus dem Jahr 2008 mit dem Satz: „Um ein sicheres Europa in einer besseren Welt aufzubauen, müssen wir mehr Einfluss auf das Geschehen nehmen. Und zwar jetzt gleich“37.

Wenn Solana - wie Algieri berichtet - die Europäische Union zur Entwicklung einer ,strategischen Kultur “38 aufruft, so soll dies einerseits die Union zu frühzeitigen, schnellen und wenn nötig robusten Kriseninterventionen befähigen. Zur strategischen Kultur gehört nach Solana aber auch die systematische Suche nach potenziellen Partnern bei der Realisierung der übergeordneten außen- und sicherheitspolitischen Zielsetzung der Europäischen Union. Solanas ausgeprägt multilateraler Außenpolitikansatz impliziert, dass er die Zukunftschancen der Europäischen Union als Ganzes eng an ihre Bereitschaft zur Zusammenarbeit mit anderen Akteuren knüpft. In der Tat konnte die Europäische Union in der Ära Solana zahlreiche ,strategische Partnerschaften mit anderen Weltregionen beziehungsweise Integrationsblöcken knüpfen. Für Solana jedoch stellten die USA und - etwas abgesetzt - Russland die wichtigsten strategischen Partner der Europäischen Union dar. Zwar leugnete der erste HV den Aufstieg der ,emerging powers' nicht, aber er betonte immer wieder die seiner Ansicht nach immer noch vorherrschende ,centrality of the US, Europe and Russia as the leading players for global security“. 39

\section{Solanas regionale Prioritäten}

„Solana's ,handwriting' became perhaps most obvious in the EU's policy towards the Western Balkans and - to a lesser degree - the Middle East conflict ${ }^{\star 40}$ analysiert Elfriede

34 So der ehemalige deutsche Außenminister Joschka Fischer zitiert nach Martin Kremer/Uwe Schmalz: Nach Nizza - Perspektiven der Gemeinsamen Europäischen Sicherheits- und Verteidigungspolitik, in: integration 2/2001, S. 167-178, hier S. 167.

35 Bernhard Stahl: The EU as a peace-making power in the Western Balkans - Solana's focal point?, in: MüllerBrandeck-Bocquet/Rüger: The High Representative for the EU Foreign and Security Policy, 2011.

36 Cristina Gallach: Preface and personal remarks, in: Müller-Brandeck-Bocquet/Rüger: The High Representative for the EU Foreign and Security Policy, 2011.

37 Rat der Europäischen Union: Bericht über die Umsetzung der Europäischen Sicherheitsstrategie - Sicherheit schaffen in einer Welt im Wandel, Dok. 17104/08.

38 Algieri: Understanding differences, 2011.

39 Solana zitiert nach ebenda.

40 Regelsberger: Treaty provisions in theory and in practice 1999-2009, 2011. 
Regelsberger. Doch obwohl Solana im Nah-Ost-Friedensprozess durch unzählige Reisen und Gespräche Entschlossenheit und Beharrlichkeit bewies, war er von den Ergebnissen seiner Mühen enttäuscht, ja frustriert. ${ }^{41}$ Enttäuschung äußerte er auch wiederholt in Bezug auf den Atomstreit mit dem Iran - in die entsprechenden Gespräche und Verhandlungen war er von der ,EU-3، (Deutschland, Frankreich und Großbritannien) erst verspätet einbezogen worden. ${ }^{42}$ Ohne jeden Zweifel aber wird die Dekade Solana auf immer mit seinem und dem Wirken der Europäischen Union auf dem Balkan verknüpft bleiben. Dazu schreibt Gallach: „It was in this region where most efforts were done and most advances were made. Naturally, the first and biggest security and defence operations were launched in the Balkans: In Bosnia and Herzegovina the EU deployed its first ever police mission [...]; in Kosovo over 3.000 EU police officers, judges and magistrates help to develop sound rule of law structures. “43 Auch Bernhard Stahl, ${ }^{44}$ der ebenfalls den westlichen Balkan als Solanas eindeutigen regionalen Schwerpunkt bezeichnet, weiß die Übernahme einer Führungsrolle durch die Europäische Union und ihr verlässliches, dauerhaftes Engagement in der Region zu würdigen. Im Detail aber führen seine Fallstudien zu einer nuancierten Gesamtbewertung mit kritischem Unterton. Denn während die Europäische Union unter ganz maßgeblichem Einfluss Solanas im Falle Mazedoniens - nicht zuletzt mittels zweier ESVP-Missionen - erfolgreich zur Stabilisierung des Landes beitragen konnte, blieb ihr im Falle der provisorischen Staaten-Union von Serbien und Montenegro der Erfolg versagt. Obwohl Solana und die Europäische Union auch hier sehr großes Engagement zeigten, ließ sich dieses Konzept letztlich nicht durchsetzen. Im Falle der EU-Aktivitäten im Kosovo fällt eine Bilanz noch schwieriger aus, da einerseits positiv das große Engagement der Europäischen Union als Staatenbauerin und Geldgeberin zu vermerken ist. Da die Europäische Union andererseits aber in der Frage der Unabhängigkeit des Kosovo keine einheitliche Position vertritt, mindert sie ihre eigene Rolle sowie die ihres HV ganz wesentlich. Denn wie soll - so Stahls rhetorische Frage - die Europäische Union glaubwürdig helfen, einen Staat aufzubauen, den nicht alle ihre Mitgliedstaaten anerkennen? Damit zeigt sich am Beispiel des Kosovo nach der Causa Irak erneut, dass Uneinigkeit oder gar Streit unter den Mitgliedstaaten die Rolle des HV ganz wesentlich beschneiden. Er kann nur dann die Europäische Union als starken internationalen Akteur ins Spiel bringen, wenn er im Namen und mit der Unterstützung aller Mitgliedstaaten sprechen und handeln kann - ist diese Voraussetzung nicht erfüllt, dann bleiben seine Waffen weitgehend stumpf. Dies lässt sich auch deutlich an der Rolle aufzeigen, die der HV bei Initiierung und Vorbereitung einer ESVP-Mission zu spielen vermag.

\section{Solana und die ESVP}

Zunächst ist erneut zu betonen, dass der 1999 gefasste Beschluss der EU-Staats- und Regierungschefs, die ESVP zu lancieren, dem HV die einzigartige Chance bot, beim Auf- und Ausbau dieser operativen Kapazitäten sowie bei der Vorbereitung und Durchführung konkreter Missionen dem neuen und wichtigen Handlungsbereich der Europäischen Union seinen Stempel aufzudrücken. Zwar ist richtig - wie Rikard Bengtsson und David Allen schreiben - „ESDP was not Solana's invention“, aber der erste HV nutzte die ihm gebotene Chance mit großen „entrepreneurial efforts“ “. ${ }^{45}$ Dazu gehörte der schon erwähnte Aufbau der

41 Gallach: Preface and personal remarks, 2011; Algieri: Understanding differences, 2011.

42 Vgl. hierzu Pierre-Emmanuel Dupont: The EU-Iran Dialogue in the Context of the Ongoing Nuclear Crisis, in: Central European Journal of International \& Security Studies 1/2009, S. 95-110.

43 Gallach: Preface and personal remarks, 2011.

44 Stahl: The EU as a peace-making power in the Western Balkans, 2011.

45 Bengtsson/Allen: Exploring a triangular drama, 2011. 
ESVP-induzierten Strukturen und Dienste. Im Folgenden hingegen soll thematisiert werden, wie der erste HV die ESVP zur Profilierung der EU-Außen- und Sicherheitspolitik nutzte. Folglich geht es hier um eine zweite Dimension von Solanas Hinterlassenschaft: seinen Beitrag zum Auftreten der Europäischen Union als operativer Akteur im Konflikt- und Krisenmanagement. Dazu Regelsberger: „Driven both by external challenges and the EU's own will to increase responsibilities in security and defense matters, the emerging ESDP became another important arena in which Solana was able to leave his mark. "46

Seinem auf Kooperation und interinstitutionelle Vernetzung setzenden Politikansatz folgend maß der erste HV den EU-NATO-Beziehungen, die zu seinem Aufgabenbereich gehörten, große Bedeutung bei. Solanas „NATO mindset" ${ }^{\star 47}$ war Garant dafür, dass er an einer möglichst reibungslosen Arbeitsteilung und Kooperation zwischen der Allianz und der ESVP interessiert war. So widmete er dem Berlin-Plus-Abkommen ${ }^{48}$ zur Zusammenarbeit zwischen NATO und ESVP, das nach komplizierten Verhandlungen im Dezember 2002 unterzeichnet werden konnte, größte Aufmerksamkeit - zum damaligen Zeitpunkt ließ sich allerdings noch nicht absehen, dass diese Regelungen über die militärische Kooperation von NATO und Europäischer Union wegen des anhaltenden Konflikts zwischen der Türkei und Zypern nur zwei Mal, nämlich im Falle der Missionen „Concordia“ und „Althea“ angewandt werden konnten.

Solana war - wiederum wohl aufgrund seines „NATO mindset“449 - zuvörderst an dem militärischen Aspekt der ESVP interessiert. Dies bringt ihm den Vorwurf Hylke Dijkstras ein, den Aufbau ziviler ESVP-Krisenreaktionskapazitäten unnötig verzögert zu haben. ${ }^{50}$ Demgegenüber betont Algieri, dass Solana stets Wert auf ,well tailored instruments comprising both civilian and military means" ${ }^{" 51}$ gelegt und eine ausgewogene Balance beider Ansätze verfolgt habe mit dem Ziel, die Europäische Union zu einer einzigartigen Krisenmanagerin zu machen. „Establishing a military capability is an important element of a properly functioning CFSP. [...] If we are serious about creating military capabilities, as well as enhancing existing civilian capabilities, the EU will be able to play a unique role across the full range of humanitarian and peacekeeping tasks. "52 Auch Gallach betont, dass der erste HV sich durchaus des Alleinstellungsmerkmals des doppelten zivil-militärischen Ansatzes der EU-Kriseninterventionskapazitäten bewusst war. ${ }^{53}$ Den Mehrwert des europäischen Ansatzes - gerade in Abgrenzung zu den USA und zur NATO - herauszustreichen, entspricht eindeutig den europäischen Interessen. Daher habe Solana ihn dezidiert vertreten. ${ }^{54}$ So sprach Solana von der Europäischen Union als ,,a global provider for security “55 und meinte damit sowohl die militärischen als auch die zivilen Interventionskapazitäten. Wenn Solana dennoch eine zu starke Betonung der militärischen Dimension der ESVP unterstellt wird, ${ }^{56}$ so

46 Regelsberger: Treaty Provisions in theory and in practice 1999-2009, 2011.

47 Algieri: Understanding differences, 2011.

48 Vgl. hierzu Martin Reichard: Some Legal Issues Concerning the EU-NATO Berlin Plus Agreement, in: Nordic Journal of International Law 1/2004, S. 37-67.

49 Algieri: Understanding differences, 2011.

50 Dijkstra: Solana and his Civil Servants, 2011.

51 Algieri: Understanding differences, 2011

52 Solana zitiert nach ebenda.

53 Gallach: Preface and personal remarks, 2011.

54 Hintergrundgespräche am 18.03.2010 in Brüssel.

55 Solana zitiert nach Algieri: Understanding differences, 2011.

56 Wegen dieser ihm zugeschriebenen Militarisierung der europäischen Außenpolitik (und wegen seiner Beteiligung als NATO-Generalsekretär am Kosovo-Krieg) erhoben sich Proteste, als Solana 2007 den Karlspreis erhielt. Vgl. die Kampagne „Not in our name!“ unter: http://www.kein-karlspreis-an-solana.de (letzter Zugriff: 07.01.2011). 
ist daran zu erinnern, dass angesichts der jahrzehntelangen sicherheitspolitischen Abstinenz von Europäischer Gemeinschaft beziehungsweise Europäischer Union das wichtigste Novum und der größte Mehrwert der ESVP-Beschlüsse des Jahres 1999 in der neuen Bereitschaft der Europäischen Union bestanden, notfalls auch militärisch in Krisen und Konflikte einzugreifen. Exakt an diesem politischen Willen hatte es allzu lange gefehlt.

Was nun konkret die Rolle, den Einfluss und den Gestaltungsspielraum anbelangt, die der erste HV beim Initiieren und Lancieren von ESVP-Missionen zu spielen vermochte, so ist festzuhalten, dass diese insgesamt recht beschränkt waren. ${ }^{57}$ Wie bereits erwähnt übte Solana im Falle der militärischen ESVP-Mission „Artemis“ 2003 in der Demokratischen Republik Kongo keine Initiativrolle aus. Vielmehr war es Frankreich, das in Absprache mit dem damaligen Generalsekretär der Vereinten Nationen Kofi Annan das Vorhaben einer militärischen Krisenintervention vorantrieb. Dadurch allerdings eröffnete sich Solana dann die Möglichkeit, den Einsatz zu realisieren. Hier erwiesen sich auch die Dienste des HV von großer Nützlichkeit: „,They enabled Solana to develop proposals, to inject them into the European structures and defend them. ${ }^{\circ 58}$ Am konkreten Beschluss, die Mission zu entsenden, war der HV jedoch vertragsgemäß nicht beteiligt.

Zusammenfassend lässt sich Solanas Rolle beim Initiieren und Lancieren von militärischen ESVP-Missionen am besten beschreiben als ,that of a facilitator, point of contact for diplomatic relations, and moderator“"59. Hier ist zu ergänzen, dass Solanas Rolle im Falle von zivilen Einsätzen durchaus bedeutsamer sein konnte, da diese weniger prominent und kontrovers debattiert und aus dem Gemeinschaftshaushalt finanziert werden, wohingegen militärische Missionen nach dem NATO-Prinzip ,,costs lie where they fall“ zu Lasten der teilnehmenden Mitgliedstaaten gehen. ${ }^{60}$

Die ersten autonomen militärischen Einsätze der Europäischen Union fanden auf dem afrikanischen Kontinent statt. ${ }^{61}$ Daraus lässt sich aber nun keineswegs schließen, dass Solana dem Nachbarkontinent eine besonders große Priorität einräumte. Vielmehr lässt sich argumentieren, dass Solana und die Mitgliedstaaten die Krisen und Konflikte in SubsaharaAfrika nutzten, um vor Ort Europas Handlungsfähigkeit und -willigkeit zu demonstrieren zumal im Kontext der in der Irak-Frage zutiefst zerstrittenen Europäischen Union.

\section{Solanas Erfolgsgeschichte - zwei Relativierungen}

Solanas Wirken als erster HV der Europäischen Union kann trotz aller Begrenzungen seines Amtes und seiner Rolle als eine „,success story“ ${ }^{“ 62}$ bezeichnet werden. Im Zentrum dieser Erfolgsbilanz Solanas steht die Tatsache, dass er der Europäischen Union auf dem internationalen Parkett Gesicht und Stimme verlieh und als Personifizierung der neuen operativen Handlungsfähigkeit der Europäischen Union wahrgenommen wurde. Insofern konnte er die internationale Rolle der Europäischen Union tatsächlich stärken.

An diesem positiven Bild sind jedoch Abstriche beziehungsweise Relativierungen vorzunehmen. So ist zum einen anzumerken, dass Solanas Engagement für die EU-Außen- und Sicherheitspolitik deutlichen zeitlichen Schwankungen unterlag. Denn während HV Solana unmittelbar nach seiner Ernennung eine ungeheure Energie an den Tag legte, um sein Amt

57 Major: Pulling the strings behind the scenes, 2011.

58 Ebenda.

59 Ebenda.

60 Ebenda.

61 Autonome militärische ESVP-Einsätze greifen nicht auf NATO-Ressourcen zurück. Sie stehen folglich außerhalb des Berlin-Plus-Abkommens.

62 Regelsberger: Treaty provisions in theory and in practice 1999-2009, 2011. 
auszufüllen und zu profilieren sowie angesichts zahlreicher Krisen und Konflikte im Namen der Europäischen Union Flagge zu zeigen, ${ }^{63}$ so lässt sich spätestens ab 2005 eine deutliche Desillusionierung beobachten, die sich in ,,indications of decreasing energy on the side of Solana in the latter part of his tenure" niederschlugen. ${ }^{64}$ Ursächlich hierfür war vorrangig, dass nach den negativen Referenden über den Verfassungsvertrag in Frankreich und den Niederlanden im Frühsommer 2005 sich Solanas Traum und Ambition, der erste Außenminister der Europäischen Union zu werden, zerschlugen. Dies setzte seinen beruflichen Ambitionen ein definitives Ende und schwächte seine Position als HV merklich, sodass auch seine größten Gegenspieler, die Mitgliedstaaten, ,,an increasing tendency“ an den Tag legten, ,to bypass him“. 65

Zweitens darf nicht vergessen werden, dass Solanas Amtsantritt in eine für sein Amt und seine Aufgaben äußerst günstige Zeitspanne fiel. Denn angesichts des Versagens der Europäischen Union in Jugoslawien Anfang/Mitte der 1990er Jahre, erneut Ende des Jahrzehnts im Kosovo und angesichts des wachsenden Drucks der USA, Europa möge in seinem Hinterhof selbst für Ordnung sorgen, entschlossen sich die EU-Staats- und Regierungschefs zum Einstieg in die ESVP. Aus dieser neuen Entschlossenheit der Europäischen Union, die von Politikern wie Tony Blair, Jacques Chirac und Gerhard Schröder ausging, ergab sich für Solana eine große Chance und für GASP und ESVP eine, goldene' Zeit, die allerdings nur kurz währte. Dass der erste HV diese Chance, die sich - eine typisch europäische Paradoxie - aus den Europa umgebenden Krisen und Konflikten ergab, umgehend und intensiv nutzte, steht ein weiteres Mal für seine Verdienste um Europas internationale Rolle. Das günstige Zeitfenster konnte aber nicht er, sondern allein die Mitgliedstaaten aufstoßen, die ihm somit einen positiven und erfolgsträchtigen Aktionskontext anboten.

Als Solana 2007 für seine Verdienste um Europa der Internationale Karlspreis zu Aachen verliehen wurde, rühmte sein Laudator, das europäische Urgestein Jean-Claude Juncker: „Nach Solana wird nichts mehr in der Welt ohne Europa gehen, wenn es um wichtige Dinge geht, und darum gebührt ihm die allergrößte Anerkennung all derer, die Wert darauf legen, dass Europa vom Rest der Welt wahrgenommen wird." 66

Doch das goldene Zeitalter für Europas Außen- und Sicherheitspolitik währte nur kurz. Machtwechsel in verschiedenen Hauptstädten sowie das Auftreten der alle Energien absorbierenden Wirtschafts- und Finanzkrise ab 2008 haben das identitätsstiftende Projekt, Europa zu einer internationalen Friedensmacht auszubauen, anscheinend wieder in den Hintergrund treten lassen. Daher musste Solanas Nachfolgerin, Ashton, ihr Amt in einem für GASP und Gemeinsame Sicherheits- und Verteidigungspolitik (GSVP) wesentlich ungünstigeren Kontext antreten.

\section{,Lessons learned': der neue Hohe Vertreter und die EU-Außen- und Sicherheitspoli- tik nach Lissabon}

Der Vertrag von Lissabon hat das Amt des HV substanziell ausgebaut und aufgewertet. Allein diese Tatsache kann als Beweis dafür gewertet werden, dass die 27 Mitgliedstaaten das Wirken des ersten HV schätzen gelernt haben und die Funktion als nützlich sowie ausbauwürdig erachten. Im Folgenden soll ein knapper Blick auf die neuen primärrechtlichen Grundlagen des Amtes geworfen werden. Weiterhin ist der Stand des Aufbaus des EAD zu

63 Duke: Under the Authority of the High Representative, 2011; Dijkstra: Solana and his Civil Servants, 2011.

64 Bengtsson/Allen: Exploring a triangular drama, 2011.

65 Ebenda.

66 Jean-Claude Juncker: Laudatio auf den Karlspreisträger 2007, Aachen, 17.05.2007. 
thematisieren. Welche Schlussfolgerungen kann man aus einem Jahr Lissabon-Praxis ziehen? Konnte die neue HV Ashton der EU-Außen- und Sicherheitspolitik bereits ihren Stempel aufdrücken? In welchem politischen Umfeld müssen sie und ihre neuen Dienste agieren? Oder anders formuliert: Wie ist es derzeit um den politischen Willen der Europäischen Union und ihrer Mitgliedstaaten bestellt, die außen- und sicherheitspolitische Akteursqualität der Union zu stärken und ihre internationale Rolle weiter auszubauen?

\section{Der „HV 2.0“ im Vertrag von Lissabon}

Der Vertrag von Lissabon hebt die EU-Außen- und Sicherheitspolitik auf eine ,qualitativ neue Stufe“67. Der „HV 2.0“68 bildet das Kernstück der institutionellen Reformen in diesem Bereich. Lässt man den langen Weg von ,Laeken“ bis ,Lissabon“ Revue passieren, ${ }^{69}$ so ist festzuhalten, dass die Substanz des sogenannten Doppelhutmodells den äußerst mühsamen und turbulenten Reformprozess unbeschadet überstanden hat. Ein Vergleich des dem neuen Amt gewidmeten Art. 18 EUV mit Art. I-28 Verfassungsvertrag belegt, dass hier keinerlei inhaltliche Änderungen vorgenommen wurden. Allerdings musste auf Drängen gewisser Mitgliedstaaten, die einen angeblichen europäischen Superstaat meinen verhindern zu müssen, der ursprüngliche, eingängige Titel „Außenminister der Union“ durch die deutlich unattraktivere Bezeichnung „Hoher Vertreter der Union für Außen- und Sicherheitspolitik“ ersetzt werden.

Mit der in Art. 18 EUV festgeschriebenen Fusion der Posten des HV und des Außenkommissars zu einem ,noch Höheren Vertreter“70 sollte einer Reihe seit Langem anerkannter Defizite der EU-Außenbeziehungen Abhilfe geschaffen werden. So beabsichtigten die Vertragsarchitekten, mit dem neu konstruierten Amt insbesondere zwei strukturelle Schwachstellen zu beheben: Zum einen sollte das doppelte Kohärenzdefizit ${ }^{71}$ der EU-Außenpolitik beseitigt werden. Das erste, horizontale Kohärenzdefizit beruht auf den unterschiedlichen Entwicklungspfaden der europäischen Politikbereiche mit externem Bezug, also auf der Mehrdimensionalität des EU-Außenhandelns. So wurden die entsprechenden Zuständigkeiten auf zwei unterschiedliche Säulen und mithin auf die Sphäre der Kommission sowie die des Rates aufgeteilt. Während erstere durch den Außenkommissar repräsentiert wurde, vertrat der HV die Ratsseite - eine Konstruktion, die Säulen übergreifende, interinstitutionelle Kohärenz tendenziell behinderte. Die Zusammenlegung beider Posten im Vertrag von Lissabon soll folglich die horizontale Kohärenz stärken und Synergien freisetzen - kurzum: europäische Außenpolitik aus einem Guss garantieren. Der neue HV ist zudem Vorsitzender im Rat Auswärtige Angelegenheiten ${ }^{72}$ sowie Vizepräsident der Kommission. Innerhalb des Kollegiums ist er für Außenbeziehungen zuständig und nur in dieser Eigenschaft dem Zustimmungsvotum des Europäischen Parlaments unterworfen. ${ }^{73}$ Somit stellt er eine instituti-

67 Elfriede Regelsberger: Von Nizza nach Lissabon - das neue konstitutionelle Angebot für die Gemeinsame AuBen- und Sicherheitspolitik der EU, in: integration 3/2008, S. 266-280, hier S. 266.

68 Rüger: A position under construction, 2011.

69 Vgl. Gisela Müller-Brandeck-Bocquet: Deutsche Europapolitik. Von Adenauer bis Merkel, Wiesbaden 2010, S. 195-211 und 270-290; Hartmut Marhold: Von der Währungsunion zur (gescheiterten) Konstitutionalisierung der Europäischen Union - zehn Jahre Systemreform, ein Kapitel Integrationsgeschichte (1999-2008), in diesem Band, S. 3-23.

70 So die scherzhafte Bezeichnung des neuen Amtes durch den ehemaligen Außenkommissar Patten. Vgl. Patten: Not quite the diplomat, 2006, S. 160.

71 Vgl. hierzu auch Simon Nuttall: Coherence and Consistency, in: Christopher Hill/Michael Smith (Hrsg.): The European Union and International Relations, Oxford 2005, S. 91-112, hier S. 97.

72 Art. 18 Abs. 3 EUV.

73 Art. 18 Abs. 4 EUV. 
onelle Zwitterkonstruktion mit hybridem Charakter dar, die die auswärtigen Politikbereiche der supranationalen und der intergouvernementalen Sphäre verbindet.

Weiterhin soll der neue HV auch die vertikale Kohärenz, das heißt die Kohärenz zwischen der Ebene der Mitgliedstaaten und der der Union, gewährleisten und dafür Sorge tragen, dass die Mitgliedstaaten ,die Außen- und Sicherheitspolitik der Union aktiv und vorbehaltlos im Geist der Loyalität und der gegenseitigen Solidarität“ unterstützen, ,das Handeln der Union in diesem Bereich“74 achten und ,sich jeder Handlung, die den Interessen der Union zuwiderläuft oder ihrer Wirksamkeit als kohärente Kraft in den internationalen Beziehungen schaden könnte" 75 enthalten. Wie dem bisher für die vertikale Kohärenz zuständigen Rat fehlen allerdings auch dem nun verantwortlichen HV jegliche Sanktionsmöglichkeiten, sollte ein Mitgliedstaat gegen diese Norm verstoßen.

Zum anderen stellt das Sichtbarkeitsproblem der Europäischen Union auf globaler Bühne einen strukturell bedingten Schwachpunkt ihrer Außenpolitik dar. Die Europäische Union wird von vielen (zu vielen?) Persönlichkeiten vertreten. Javier Solana als ihr Gesicht und ihre Stimme konnte das Problem nur lindern, nicht lösen. Mit dem Lissabonner Doppelhut - der eigentlich ein ,dreifacher Hut ${ }^{\star}$ ist, weil in ihm die früheren Funktionen des HV, des Außenkommissars sowie der rotierenden Ratspräsidentschaft im Bereich Außen- und Sicherheitspolitik verschmolzen sind - hofft man, die vielgestaltigen Erscheinungsformen der Union auf internationalem Parkett zu überwinden. Als „Troika in einer Person“76, die das bisherige europäische Dreiergespann ablöst, soll der „HV 2.0“ die externe Wahrnehmbarkeit der Europäischen Union substanziell erhöhen. Auch die mindestens fünfjährige Amtszeit, die vorrangig die Kontinuität der EU-Außenpolitik befördern soll, wird das Sichtbarkeitsproblem lindern.

Wie stark das neue Primärrecht das Amt des HV aufwertet, wird klar, wenn man die bescheidene Amsterdamer Vertragsgrundlage zum Vergleich nimmt. Rein quantitativ fällt auf, dass der Begriff Hoher Vertreter im Vertrag von Lissabon 72-mal fällt, im Vertrag von Amsterdam hingegen nur ganze dreimal. Wichtiger aber ist die qualitative Aufwertung: Von einer die Präsidentschaften unterstützenden Position im EUV-Amsterdam wurde das Amt des HV zu einem veritablen Manager des EU-Außenhandelns aufgewertet. So umfassen die Zuständigkeiten des „HV 2.0“ heute das Initiativrecht, Koordinierungs-, Kontroll- und Umsetzungsbefugnisse, Aufgaben in der Konfliktprävention und im Krisenmanagement sowie die generelle Außenvertretung der Union auf seiner Ebene. Der „HV 2.0“ ist demnach Koordinator, Motor, Vermittler, Kontrollinstanz und Sprecher der Union - kurzum Dreh- und Angelpunkt der EU-Außen- und Sicherheitspolitik in ihrer Gesamtheit. ${ }^{77}$

Obwohl der Vertrag - wie aufgezeigt - das Potenzial zur Abmilderung der bekannten Defizite mit sich bringt, sind Zweifel angebracht, ob der „HV 2.0“ den hohen Erwartungen in der Praxis gerecht werden kann. Anders als vielfach behauptet, sind diese Zweifel nicht primär in der persönlichen und fachlichen Qualifikation der ersten Amtsinhaberin begründet, sondern hängen direkt mit der Konstruktion der neuen Position zusammen, aus der sich vorrangig zwei Dilemmata ergeben: Erstens kann der strukturell gewollte Balanceakt zwischen zwei institutionellen Sphären leicht dazu führen, dass der „HV 2.0“ aus dem Gleichgewicht und zwischen die Fronten gerät. ,A double-hatted High Representative always bears the risk of becoming , double-hated'."78 Zweitens geht der Doppelhut auch mit einer doppelten Arbeitsbelastung einher - einer dreifachen gar, wenn man den ständigen Vorsitz im Außenmi-

74 Art. 24 Abs. 3 EUV.

75 Ebenda.

76 Rüger: A position under construction, 2011.

77 Vgl. Art. 15, 17, 18, 21, 22, 24, 26, 27, 30, 31, 32, 33, 34, 36, 38, 41, 42, 43, 44, 46 EUV.

78 Rüger: A position under construction, 2011. 
nisterrat hinzuzählt. Angesichts dieser Aufgabenfülle und der Tatsache, dass die Pflichten nicht nur in Brüssel, sondern weltweit wahrgenommen werden müssen - Solana hat angeblich vier Millionen Reisekilometer im Dienst der Europäischen Union zurückgelegt -, ist nicht verwunderlich, dass der Posten bereits als „mission impossible“ verrufen wird. Bezeichnenderweise gehören die früheren Amtsinhaber Solana und Patten zu den Kritikern des Doppelhuts. Dies taten sie während der Reformdebatten kund. Solana äußerte explizit die Befürchtung, die Ämterfusion würde zu ,,mehr Verwirrung als Synergie“79 führen. Da die Mitgliedstaaten die Verschmelzung der Ämter jedoch befürworteten, verzichtete er auf Widerstand. ${ }^{80}$

\section{Der Aufbau des EAD - , einmalige Gelegenheit‘ oder institutionelles Schlachtfeld?}

Wie schwierig - um nicht zu sagen unmöglich - es ist, die Aufgaben des „HV 2.0“ zu allseitiger Zufriedenheit auszufüllen, kann exemplarisch an der umkämpften Einrichtung des EAD gezeigt werden, Ashtons erstem großen Lackmustest. Die neue HV selbst hatte die Messlatte sehr hoch gehängt, indem sie das Ziel proklamierte, den EAD zum ,,pride of Europe and the envy of the rest of the world" ${ }^{\circ 1}$ machen zu wollen. An anderer Stelle sprach sie von einer „once-in-a-generation opportunity“. 82

Während es bislang - anders als allgemein erwartet - zu keinen nennenswerten Konkurrenzkämpfen zwischen Ashton und dem ständigen Präsidenten des Europäischen Rates, Herman Van Rompuy, kam, geriet der Aufbau des EAD zu einem monatelangen institutionellen ,Hauen und Stechen' zwischen den Hauptakteuren. ${ }^{83}$ Sie alle (Rat/Mitgliedstaaten, Kommission, Europäisches Parlament) machten ihre Ansprüche geltend und versuchten, die eigenen Einflusssphären möglichst großzügig abzustecken. Dabei leisteten die sehr vagen Bestimmungen des einschlägigen Art. 27 Abs. 3 EUV diesen Kämpfen um Macht und Einfluss im neuen EAD Vorschub. Doch statt diese vertragsrechtliche Offenheit konstruktiv zu nutzen, verlegten sich die Akteure auf ,defensive Verhandlungsstrategien“84. Dies gilt insbesondere für die Mitgliedstaaten, die sich nur zu nachrangigen Fragen wie Sprachregelung oder Länderquoten zu Wort meldeten, strategische Vorschläge jedoch vermissen ließen.

Der EAD, der zum 1. Dezember 2010 seine Tätigkeit aufnahm, ist eine Behörde sui generis zwischen Kommission und Ratssekretariat, die unter der Verantwortung der HV steht, und gleicht weitgehend einem klassischen Außenministerium. Der derzeit rund 3.700 Mitarbeiter (darunter viele aus den Vertretungen der Kommission sowie aus den ,Solana-Strukturen') zählende Dienst wird in den kommenden Jahren substanziell ausgebaut. Doch schon in

79 The European Convention. The Secretariat: Address of the Secretary-General/High Representative Javier Solana at the meeting of WG VII on 15 October 2002, Working Group VII, Working Document 8, 15.10.2002, Brussels.

80 So Christina Gallach im Hintergrundgespräch am 18.03.2010 in Brüssel.

81 Ashton in ihrer schriftlichen Antwort auf den Fragebogen des Europäischen Parlaments zur Anhörung der designierten Kommissare. Europäisches Parlament, Ausschuss für Auswärtige Angelegenheiten: Mitteilung an die Mitglieder. Anhörung von Baroness Ashton, designierte Vizepräsidentin der Kommission/Hohe Vertreterin der Union für Außen- und Sicherheitspolitik, Dok. PE431.071v02-00.

82 Vgl. Catherine Ashton: Rede auf der 46. Münchner Sicherheitskonferenz, 06.02.2010, abrufbar unter: http:// www.securityconference.de/Ashton-Catherine.567.0.html (letzter Zugriff: 07.01.2011).

83 Für eine umfassende Übersicht zum EAD vgl. Julia Lieb/Martin Kremer: Der Aufbau des Europäischen Auswärtigen Dienstes: Stand und Perspektiven, in: integration 3/2010, S. 195-208; Cameron: The EU's External Action Service, 2011; Rüger: A position under construction, 2011 sowie Gisela Müller-Brandeck-Bocquet/Carolin Rüger: The legacy of Javier Solana, the High Representative 2.0 and the European External Action Service: strong foundations for the EU's international role?, in: Müller-Brandeck-Bocquet/Rüger: The High Representative for the EU Foreign and Security Policy, 2011.

84 Lieb/Kremer: Der Aufbau des Europäischen Auswärtigen Dienstes, 2010, S. 199. 
naher Zukunft wird sich zeigen, ob dieses Gesellenstück Ashtons sich in der Praxis bewährt und zur Stärkung der EU-Außenbeziehungen beiträgt.

\section{Mission impossible? HV Catherine Ashton nach ihrem ersten Amtsjahr}

Die Reaktionen auf die Ernennung der politischen „Leichtgewichte“ Van Rompuy zum ständigen Präsidenten des Europäischen Rates ${ }^{85}$ und Ashton zur HV am 19. November 2009 reichten von großem Erstaunen bis zu offenen Missfallensbekundungen. Es wurde gar von einer „Selbstverzwergung" 86 der Europäischen Union gesprochen. Insbesondere Ashton schlug von Beginn an heftiges Misstrauen entgegen, wie die wenig schmeichelhaften Titulierungen „Aschenputtel mit Jetlag“87, „Lady Who“ ${ }^{\circ 8}$ oder „,bange Baroness" ${ }^{\circ 9}$ belegen. Ihre Autorität litt zudem dadurch Schaden, dass Kommissionspräsident Barroso die Zuständigkeit für die Europäische Nachbarschaftspolitik (ENP), die bislang zum Portfolio des Außenkommissars gehörte, Ashton entzog und dem Verantwortungsbereich des Erweiterungskommissars zuwies. Machtbewusst stellte Barroso damit zwar sicher, dass die ENP mit ihren beträchtlichen Finanzmitteln in der Kommissionssphäre verblieb, beraubte gleichzeitig jedoch die neue HV und Vize-Kommissionspräsidentin einer idealen Möglichkeit zur Nutzbarmachung ihres Doppelhuts und zur Gewährleistung der institutionellen Kohärenz, die in der ENP von Nöten ist.

In der breiteren Öffentlichkeit wurde Ashton insbesondere vorgeworfen, dass sie als auBenpolitische Novizin mit wenig Erfahrung auf dem internationalen diplomatischen Parkett ihre Ernennung allein der Tatsache verdanke, die Kriterien ,Frau, britisch, Mitte-Links ' erfüllt zu haben. Die harsche Kritik anlässlich der Ernennung der neuen HV ebbte auch nach deren Amtsantritt nicht ab: Ihre ersten Auftritte vor dem Europäischen Parlament Ende 2009 und Anfang 2010 wurden kühl aufgenommen und die Wahl ihrer Auswärtstermine heftig kritisiert. Auch Ashtons Agieren beim Aufbau des EAD bot Anlass zu vielstimmiger Kritik. Als ihre Wahl für den prestigeträchtigen Posten des EU-Delegationsleiters in Washington Mitte 2010 auf einen engen Vertrauten von Kommissionspräsident Barroso fiel, gab es erneut Schelte. Und Anfang Dezember 2010 bezog erstmals Ashtons Vorgänger offen Position gegen die neue HV: In einem Brief forderte Solana zusammen mit zahlreichen anderen namhaften Persönlichkeiten wie Chris Patten, Romano Prodi, Felipe González, Richard von Weizsäcker oder Helmut Schmidt ein härteres Vorgehen der Europäischen Union gegen Israel. ${ }^{90}$ Dieser Kritikflut steht als erster und bisher einziger inhaltlicher Erfolg Ashtons eine Resolution der Vereinten Nationen vom September $2010^{91}$ gegenüber, die den Weg für direkte Gespräche zwischen Serbien und dem Kosovo ebnet. Das unerwartete Einlenken des serbischen Präsidenten Boris Tadić wird dem Verhandlungsgeschick der HV zugeschrieben.

Insgesamt ist die an Ashton geäußerte Kritik zu relativieren. Allein schon die Klagen über ihre angeblich gänzlich fehlende außenpolitische Kompetenz waren angesichts ihrer Tätigkeit als Außenhandelskommissarin überzogen, hatte sie doch beispielsweise das Freihandelsabkommen mit Südkorea, das wichtigste derartige Abkommen überhaupt, erfolg-

85 Vgl. zu diesem Amt Wolfgang Wessels/Thomas Traguth: Der hauptamtliche Präsident des Europäischen Rates: „Herr“ oder „Diener“ im Haus Europa?, in: integration 4/2010, S. 297-311.

86 Die Welt: Europas Selbstverzwergung schockt die USA, 21.11.2009.

87 Walter Mayr: Aschenputtel mit Jetlag, in: Der Spiegel, 08.03.2010.

88 Handelsblatt: Lady Who und ihre schwierige Mission, 16.03.2010.

89 Claus Hecking/Peter Ehrlich: Die bange Baroness Ashton, in: Financial Times Deutschland, 02.02.2010.

90 Vgl. Andrew Rettmann: Former EU leaders challenge Ashton on Israel, in: euobserver.com, 10.12.2010.

91 United Nations. General Assembly: Request for an advisory opinion of the International Court of Justice on whether the unilateral declaration of independence of Kosovo is in accordance with international law, Resolution adopted by the General Assembly, A/RES/64/298. 
reich abgeschlossen. Und auch Ashtons Absenz bei hochrangigen Treffen war kein Zeichen von Ignoranz, sondern einem übervollen Terminkalender geschuldet. So zeigt sich schon nach einem Jahr der Amtsführung der „HV 2.0“, dass die Warnungen Solanas und Pattens vor einer allzu hohen, de facto nicht bewältigbaren Arbeitsbelastung der doppelhütigen Vertreterin von der Realität bestätigt wurden - das Amt scheint in der Tat eine „mission impossible “92 zu sein.

Nachdem die HV Ashton in ihrem ersten Amtsjahr sich weitgehend dem kräftezehrenden Aufbau des EAD gewidmet und die inhaltliche Profilbildung für Europas Außenpolitik deutlich vernachlässigt hatte, scheint sie seit Kurzem dieses Defizit beheben zu wollen. In ihrem ersten neunzehnseitigen Sachstandsbericht, den sie dem Europäischen Rat am 17. Dezember 2010 vorlegte und der die Beziehungen mit China, Russland und den USA in den Blick nimmt, lässt sich eine sehr pragmatische Herangehensweise ablesen. So nimmt das Papier zwar auf Menschenrechtsbelange Bezug, Ashton plädiert jedoch für eine Aufhebung des Waffenembargos gegen die Volksrepublik und konstatiert: „China will not match EU standards of human rights and rule of law for some time to come. Future convergence is best sought by concentrating on common ground." "93 Für Russland sieht Ashton die Priorität dagegen in der Beschleunigung der demokratischen Transition. Und mit Blick auf die USA zeigt sie selbstkritischen Realismus in Bezug auf Europas Auftreten: „If we over-promise and under-deliver; if we prioritise process over substance or if we don't know what we want, the US will turn its attention elsewhere. “94 Ein weiteres Strategiepapier für die Beziehungen mit Brasilien, Indien und Südafrika ist bereits angekündigt.

\section{Ausblick}

Sachstandsberichte und Strategiepapiere sind das eine - wirksames Auftreten im Namen der Europäischen Union das andere. Ashton hat also noch viel Bewegungsspielraum nach oben, um das Amt des „HV 2.0“ vollumfänglich und im Sinne der Stärkung der internationalen Rolle der Europäischen Union auszufüllen. Doch wie es auch bei ihrem Amtsvorgänger der Fall war, wird Ashtons Aktionsspielraum und ihre Rolle in großem Maße von der Entschlossenheit und dem politischen Willen der Mitgliedstaaten, die Europäische Union zu einem zentralen Akteur der Weltpolitik auszubauen, abhängen. Und daran scheint es derzeit gravierend zu mangeln. Die Staats- und Regierungschefs der von Finanz-, Wirtschafts- und Eurokrise geschüttelten Europäischen Union verausgaben sich derzeit im kurzfristigen Krisenmanagement und lassen jegliche Blaupause für die Zukunft der Europäischen Union vermissen. Jüngst haben hochrangige Persönlichkeiten in einem Appell diese Visionslosigkeit der aktuellen europäischen Führungsriege beklagt und Veränderungen angemahnt. ${ }^{95}$ Der gemeinsame Brief, den Deutschland, Frankreich und Polen am 15. Dezember 2010 an die HV Ashton schrieben, bietet hier eventuell einen ersten Ansatzpunkt. Die Außen- und Verteidigungsminister des Weimarer Dreiecks klagen darin einen „frischen Impuls“ für die weitgehend zum Erliegen gekommene ESVP ein. ${ }^{96}$ Und auch eine deutsch-schwedische Initiative,

92 Philipp Ricard/Jean-Pierre Stroobants: Lady Ashton - mission impossible, in: Le Monde, 28.01.2011; Martin Winter: Auf Bewährung. Zögerlich und unerfahren: Die EU-Außenbeauftragte Catherine Ashton kämpft ein Jahr nach Amtsantritt noch immer mit ihrem schlechten Ruf, in: Süddeutsche Zeitung, 19./20.02.2011.

93 Ashton zitiert nach Andrew Rettmann: Ashton pragmatic on China in EU foreign policy blueprint, in: euobsorver.com, 17.12.2010.

94 Ebenda.

95 Das Manifest der im September 2010 neu gegründeten Spinelli-Gruppe ist abrufbar unter: http://www.spinelligroup.eu (letzter Zugriff: 07.01.2011).

96 Martin Winter: Mühselige Verhandlungen. EU-Länder wollen Sicherheitspolitik verbessern, in: Süddeutsche Zeitung, 15.12.2010. 
ebenfalls von Ende 2010, fordert alle EU-Mitgliedstaaten dazu auf, die Synergien zu identifizieren, die sich durch stärkere Zusammenarbeit in den Verteidigungsstrukturen ergeben könnten. Ob Ashton, der nur geringes Interesse an der GSVP nachgesagt wird, ${ }^{97}$ daraus ein neues Momentum für die globale Rolle der Europäischen Union wird entfalten können, bleibt abzuwarten. Sicher aber ist, dass die Europäische Union einer Relance ihrer Außenund Sicherheitspolitik dringend bedarf, um die im Entstehen befindliche neue Weltordnung mitgestalten zu können. Die derzeit in Aufruhr befindliche arabische Welt erwartet dies von ihrem Nachbarn Europa.

\section{Ein konzeptioneller Leitfaden für Theorie, Empirie und Integrationspraxis}

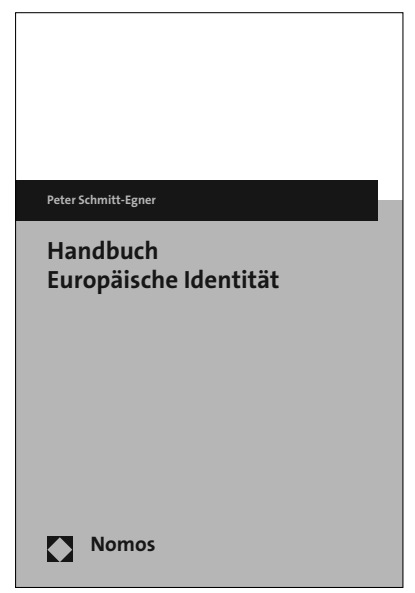

Bitte bestellen Sie im Buchhandel oder versandkostenfrei unter $\downarrow$ www.nomos-shop.de

\section{Handbuch Europäische Identität}

Von Peter Schmitt-Egner

2011, ca. 280 S., brosch., ca. 39,-€,

ISBN 978-3-8329-5229-7

Erscheint ca. August 2011

Ziel des Handbuchs ist es, einen Überblick zur politischen, wissenschaftlichen und integrationsstrategischen Dimension von Europäischer Identität zu geben. Hierzu wird ein Leitfaden in fünf Dimensionen entwickelt: politischer Entstehungs-, multidisziplinärer Begründungs-, empirischer Sach-, multidimensionaler Handlungs- und integrationstrategischer Verwendungszusammenhang.

97 Jean-Pierre Stroobants/Natalie Nougayrède: OTAN-Union européenne, le ,partenariat' reste flou, in: Le Monde, 21./22.11.2010. 